\title{
CXCL12 induces migration of oligodendrocyte precursor cells through the CXCR4-activated MEK/ERK and PI3K/AKT pathways
}

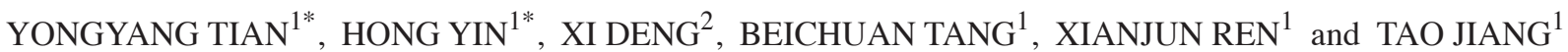 \\ Departments of ${ }^{1}$ Orthopedics and ${ }^{2}$ Ultrasound, Xinqiao Hospital, \\ Third Military Medical University (Army Medical University), Chongqing 400037, P.R. China
}

Received February 6, 2018; Accepted July 9, 2018

DOI: $10.3892 / \mathrm{mmr} .2018 .9444$

\begin{abstract}
Demyelination is a nervous system disease in which the myelin sheaths of neurons are damaged due to inflammatory reactions, inherited abnormalities or trauma. This damage impairs the conduction of signals in the affected nerves, which in turn causes deficiencies in sensation, movement and cognition. Oligodendrocyte precursor cells (OPCs) are able to induce remyelination. However, the remyelination is suboptimal due to the limited migration of OPCs. In the present study, neonatal OPCs were isolated from rats for the investigation of the role of $\mathrm{C}-\mathrm{X}-\mathrm{C}$ motif chemokine ligand 12 (CXCL12), an important chemokine, in mediating the migration ability of OPCs. The present results demonstrated that CXCL12 stimulation markedly promoted the migration of OPCs and activated the dual specificity mitogen-activated protein kinase kinase 1 (MEK)/extracellular signal-regulated kinase (ERK) and phosphoinositide 3-kinase (PI3K)/RAC- $\alpha$ serine/threonine-protein kinase (AKT) pathways. Knockdown of C-X-C motif chemokine receptor 4 (CXCR4; a receptor of CXCL12) reversed the CXCL12-induced migration of OPCs and blocked the MEK/ERK and PI3K/AKT pathways. In addition, specific inhibitors of the MEK/ERK and PI3K/AKT
\end{abstract}

Correspondence to: Professor Xianjun Ren or Dr Tao Jiang, Department of Orthopedics, Xinqiao Hospital, Third Military Medical University (Army Medical University), 83 Xinqiao Road, Chongqing 400037, P.R. China

E-mail: ren_xianjun@sina.com

E-mail: fromcq2000@sina.com

*Contributed equally

Abbreviations: CXCL12, C-X-C motif chemokine ligand 12; CXCR4, C-X-C motif chemokine receptor 4; ERK, extracellular signal-regulated kinase; PI3K, phosphoinositide 3-kinase; OPCs, oligodendrocyte precursor cells; CNS, central nervous system; PDGFR- $\alpha$, platelet-derived growth factor receptor- $\alpha$; MBP, myelin basic protein; AKT, RAC- $\alpha$ serine/threonine-protein kinase

Key words: remyelination, OPCs, CXCL12, CXCR4, ERK, AKT pathways significantly reduced the migration of OPCs. Based on these findings, it was concluded that CXCL12 may induce the migration of OPCs through the CXCR4-activated MEK/ERK and PI3K/AKT pathways. The results of the present study support the manipulation of CXCL12-mediated OPC migration to improve remyelination.

\section{Introduction}

Myelin is a fatty white substance that surrounds the axons of neurons. In the central nervous system (CNS), oligodendrocytes supply the myelin, which provides metabolic support to the axon and allows for the rapid transmission of action potentials along the axon (1). Immune responses, inherited abnormalities or trauma may result in oligodendrocyte necrosis and dysfunction, and resultant demyelination in cases of multiple sclerosis and spinal cord injury. The current treatment strategy for demyelination is based on remyelination, a process that may restore metabolic support to the axon to limit axonal degeneration and restore the nodes that are required to facilitate conduction and, therefore, function (2).

Although the CNS has little capacity for regeneration, a number of studies have demonstrated that myelin may be regenerated by oligodendrocytes (3-5). In the CNS, endogenous oligodendrocyte precursor cells (OPCs) contribute toward the replacement of oligodendrocytes required for remyelination following demyelination (6). OPCs are able to proliferate and differentiate into mature oligodendrocytes to repair injured myelin following demyelination. However, the extent and quality of endogenous remyelination is suboptimal (6). Therefore, numerous studies have aimed to promote the maturation, proliferation and differentiation of OPCs in order to improve remyelination (7-10). In addition, efforts have also been made to enhance oligodendrocyte replacement through cell transplantation by the authors of the present study and others (11-14).

Although positive results have been achieved in preclinical studies using rodent animal models, a clinical trial using human CNS stem cells that have the ability to differentiate into oligodendrocytes was performed in children with demyelination, and the result, which exhibited a modest degree of remyelination near the injection site, was far from being satisfactory (15). The different degrees of myelination between 
rodent-based preclinical studies and human-based clinical trials may be ascribed to the limited migration of the transplanted cells in the human brain (2). Notably, Boyd et al (16) reported that insufficient OPC migration into demyelinated lesions may be a critical cause of poor remyelination in multiple sclerosis. Therefore, developing effective approaches to regulate the migration of OPCs is urgently required and important for the treatment of demyelination.

C-X-C motif chemokine ligand 12 (CXCL12; formerly known as stromal cell-derived factor 1) is a well-identified chemokine that serves an important role in mediating the migration ability of multiple normal and tumor cells $(17,18)$. Previous studies have demonstrated that CXCL12 regulates the proliferation and differentiation of OPCs $(19,20)$. Notably, CXCL12 promotes the migration of OPCs and improves remyelination in vivo (20-23). However, the underlying mechanism of the CXCL12-induced migration of OPCs remains unclear. Considering that CXCL12 induces the invasion of tumors via $\mathrm{C}-\mathrm{X}-\mathrm{C}$ motif chemokine receptor 4 (CXCR4; a receptor of CXCL12) and the dual specificity mitogen-activated protein kinase kinase 1 (MEK) and phosphoinositide 3-kinase (PI3K) pathways (24-26), the present study assessed the importance of the MEK and PI3K pathways in CXCL12/CXCR4-regulated migration of OPCs.

\section{Materials and methods}

Isolation and culture of rat OPCs. The isolation and culture of OPCs was performed as previously described $(27,28)$. The cortical tissues of 8-12 neonatal Sprague-Dawley rats (postnatal day $2 ; 30$ ) were purchased from the experimental animal centre of Third Military Medical University (1:1, male: female; weight 7-10 g). The rats were housed at $25^{\circ} \mathrm{C}, 50 \%$ humidity, 12-h light/dark cycle and ad libitum access to food and water. Cortical tissues of 8-12 rats per repeat were resected to prepare a cell suspension with a $74 \mu \mathrm{M}$ filter. Following centrifugation at $200 \times \mathrm{g}$ for $10 \mathrm{~min}$ at $4^{\circ} \mathrm{C}$, the cell pellet was resuspended and cultured in a poly-lysine-coated culture flask for 10 days at $37^{\circ} \mathrm{C}$ in $5 \% \mathrm{CO}_{2}$. To purify OPCs, the flask was placed onto a rotary shaker at $180 \mathrm{rpm}$ for $1 \mathrm{~h}$. Subsequently, the supernatant in the flask was replaced with fresh OPC proliferation medium to remove the dislodged cells ( $\sim 90 \%$ microglia). Following regular culture for $2 \mathrm{~h}$, the flask was placed onto a rotary shaker at $180 \mathrm{rpm}$ for $18 \mathrm{~h}$. The following day, the supernatant was collected to pass through a cell strainer. Following centrifugation at $200 \mathrm{x} \mathrm{g}$ for $10 \mathrm{~min}$ at $4^{\circ} \mathrm{C}$, the pelleted cells were resuspended and cultured in a new flask for $1 \mathrm{~h}$. The flask was gently agitated to remove loosely adherent cells. The supernatant was collected, centrifuged at $200 \mathrm{x}$ g for $10 \mathrm{~min}$ and cultured in a new poly-lysine-coated culture flask.

The OPC proliferation medium contained Dulbecco's modified Eagle's medium (DMEM) supplemented with $0.5 \%$ fetal bovine serum (FBS), $10 \mathrm{ng} / \mathrm{ml}$ basic fibroblast growth factor, $10 \mathrm{ng} / \mathrm{ml}$ platelet-derived growth factor (PDGF)-AA, $10 \mu \mathrm{g} / \mathrm{ml}$ insulin, $30 \mathrm{nM}$ sodium selenite, $0.5 \mu \mathrm{g} / \mathrm{ml}$ transferrin, $30 \mathrm{nM}$ thyroiodine, $4 \mathrm{mM}$ L-glutamine, $5 \mathrm{mM}$ sodium pyruvate, $50 \mathrm{U} / \mathrm{ml}$ penicillin and $50 \mu \mathrm{g} / \mathrm{ml}$ streptomycin (all Invitrogen; Thermo Fisher Scientific, Inc., Waltham, MA, USA).

Differentiation of OPCs. To induce OPC differentiation, OPCs were cultured in differentiation medium for 3 days. The differentiation medium for OPCs consisted of DMEM, $0.5 \%$ FBS, $10 \mu \mathrm{g} / \mathrm{ml}$ insulin, $30 \mathrm{nM}$ sodium selenite, $0.5 \mu \mathrm{g} / \mathrm{ml}$ transferrin, $30 \mathrm{nM}$ thyroiodine, $4 \mathrm{mM}$ L-glutamine, $5 \mathrm{mM}$ sodium pyruvate, $50 \mathrm{U} / \mathrm{ml}$ penicillin and $50 \mu \mathrm{g} / \mathrm{ml}$ streptomycin (all Invitrogen; Thermo Fisher Scientific, Inc.).

Immunostaining. Cells on cover slips were fixed with $4 \%$ paraformaldehyde for $20 \mathrm{~min}$ at $4^{\circ} \mathrm{C}$, permeabilized using $0.1 \%$ Triton $\mathrm{X}-100$ for $15 \mathrm{~min}$ and blocked for $60 \mathrm{~min}$ at $23^{\circ} \mathrm{C}$ with $5 \%$ goat serum (Wuhan Boster Biological Technology, Ltd., Wuhan, China). Primary antibodies against neural/glial antigen 2 (NG2; cat. no. 14-6504-80; 1:200; Thermo Fisher Scientific, Inc.), PDGF receptor- $\alpha$ (PDGFR- $\alpha$; cat. no. sc-31178; 1:200; Santa Cruz Biotechnology, Inc., Dallas, TX, USA), oligodendrocyte marker O4 (O4; cat. no. MAB1326; 1:400; R\&D Systems, Inc., Minneapolis, MN, USA) and myelin basic protein (MBP; cat. no. sc-13526; 1:200; Santa Cruz Biotechnology, Inc.) were diluted in $5 \%$ goat serum and incubated with the samples overnight at $4^{\circ} \mathrm{C}$. The following day, the cells were incubated with secondary antibodies including goat anti-mouse $\operatorname{IgG}(\mathrm{H}+\mathrm{L})$ highly cross-adsorbed secondary antibody, Alexa Fluor Plus 488 (cat. no. A32723; Thermo Fisher Scientific, Inc.; 1:500), goat anti-mouse IgM (Heavy chain) cross-adsorbed secondary antibody, Alexa Fluor 488 (cat.no. A-21042; Thermo Fisher Scientific, Inc.; 1:500) and donkey anti-goat $\operatorname{IgG}(\mathrm{H}+\mathrm{L})$ cross-adsorbed secondary antibody, Alexa Fluor 488 (cat. no. A-11055; Thermo Fisher Scientific, Inc.; 1:400) for $1 \mathrm{~h}$ at $37^{\circ} \mathrm{C}$. The nuclei were stained with DAPI (Invitrogen; Thermo Fisher Scientific, Inc.) for $10 \mathrm{~min}$ at $23^{\circ} \mathrm{C}$. Fluorescence images were acquired using a fluorescence microscope (Olympus Corporation, Tokyo, Japan; magnification, $\mathrm{x} 200$ ).

Migration assay. The migration of OPCs was assessed using a Boyden chamber, which contained 48-well inserts with an 8- $\mu \mathrm{m}$ pore-size filter (Neuro Probe, Inc., Gaithersburg, MD, USA). A total of $5 \times 10^{4}$ OPCs were seeded in the top of the insert in proliferation medium, while different concentrations of CXCL12 (0, 5, 10 and 20 ng/ml; PeproTech, Inc., Rocky Hill, NJ, USA) diluted in proliferation medium were placed in the well below as a chemoattractant. After $48 \mathrm{~h}$, the top surface of the filter was cleared with a cotton swab. Following fixing in methanol for $20 \mathrm{~min}$ at $23^{\circ} \mathrm{C}$, the back of the filter was stained at $23^{\circ} \mathrm{C}$ for $15 \mathrm{~min}$ with $0.1 \%$ crystal violet to observe the migrated OPCs. For quantitative analysis, five random images of 480x360 $\mu \mathrm{m}$ were captured of the filter. Each group had three replicate filters. To counteract observer bias, the migrated OPCs (positive for crystal violet) were counted by an individual who was blinded to the grouping of the samples.

To test the effect of CXCR4 on the CXCL12-induced migration of OPCs, CXCR4 short hairpin RNAs (shRNAs) were used to downregulate the expression of CXCR4, while an shRNA control (shRNAcon) served as the control (methods described below). The untreated, shRNAcon-treated and CXCR4 shRNA-treated OPCs were used to perform the $20 \mathrm{ng} / \mathrm{ml} \mathrm{CXCL12-induced}$ migration assay, as described above.

To test the effect of the MEK/extracellular signal-regulated kinase (ERK) and PI3K/RAC- $\alpha$ serine/threonine-protein kinase (AKT) pathways on the CXCL12-induced migration of OPCs, OPCs were divided into four groups: OPCs; OPCs treated with $\mathrm{U} 1026$ (inhibitor of the MEK/ERK pathway; $10 \mu \mathrm{M}$; Invitrogen; 

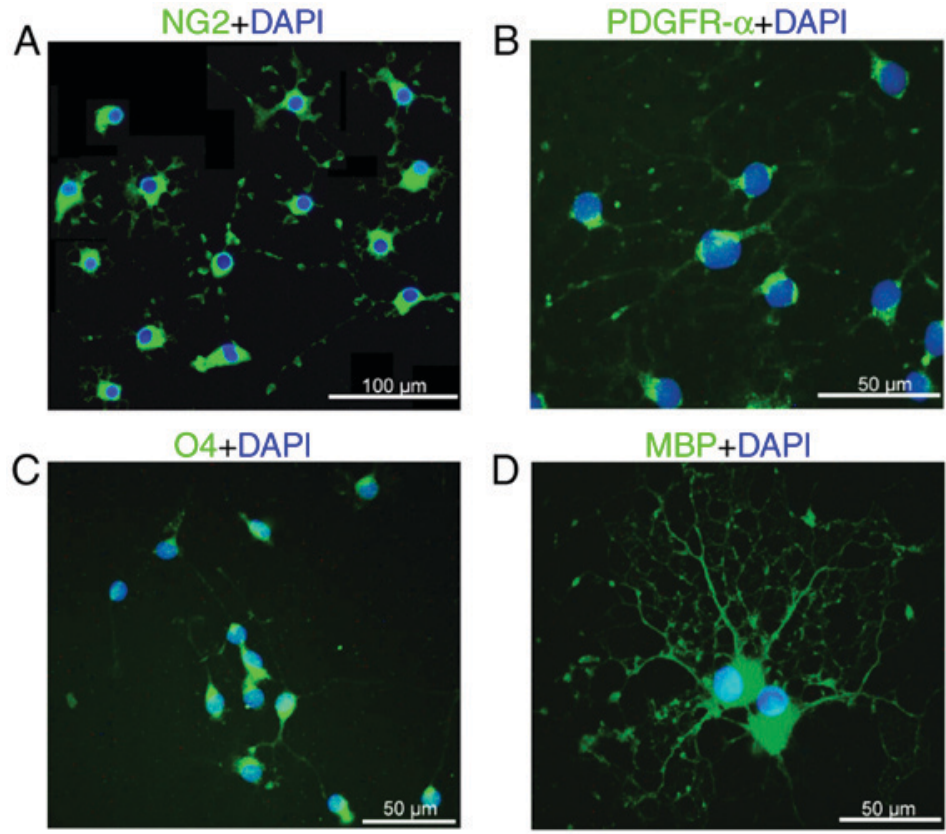

Figure 1. Isolation and identification of OPCs. (A) Immunostaining demonstrated that OPCs were positive for NG2. (B) Immunostaining demonstrated that OPCs were positive for PDGFR- $\alpha$. (C) Immunostaining showed that the differentiated OPCs were positive for O4. (D) Immunostaining showed that the differentiated OPCs were positive for MBP. Nuclei were stained with DAPI (blue). NG2, neural/glial antigen 2; PDGFR- $\alpha$, platelet-derived growth factor receptor- $\alpha$; OPCs, oligodendrocyte precursor cells; O4, oligodendrocyte marker O4; MBP, myelin basic protein.

Thermo Fisher Scientific, Inc.); OPCs treated with LY294002 (inhibitor of the PI3K/AKT pathway; $10 \mu \mathrm{M}$; Invitrogen; Thermo Fisher Scientific, Inc.); and OPCs treated with U1026 $(10 \mu \mathrm{M})$ and LY294002 (10 $\mu \mathrm{M})$. Subsequently, the four groups of OPCs were used to perform the $20 \mathrm{ng} / \mathrm{ml}$ CXCL12-induced migration assay, as described above.

Western blotting. Cells were lysed in radioimmunoprecipitation assay protein lysis buffer (Beyotime Institute of Biotechnology, Haimen, China) and centrifuged at $12,000 \mathrm{x} \mathrm{g}$ for $5 \mathrm{~min}$ at $4^{\circ} \mathrm{C}$. The supernatant was collected to determine the protein concentration using a bicinchoninic acid protein assay kit (CWBIO, Beijing, China). Total proteins (20 $\mu$ g/group) were separated using $12 \%$ SDS-PAGE and transferred onto polyvinylidene difluoride membranes (EMD Millipore, Billerica, MA, USA). Following blocking with 5\% skimmed milk in TBS with Tween-20 (TBST) for $1 \mathrm{~h}$ at $23^{\circ} \mathrm{C}$, the membranes were incubated with antibodies against CXCR4 (1:1,000; cat. no. PA1237; Wuhan Boster Biological Technology, Co., Ltd., Wuhan, China), AKT1 (1:2,000; cat. no. ab235958; Abcam, Cambridge, UK), phosphorylated (p)-AKT1 (cat. no. ab81283; 1:2,000; Abcam), ERK1/2 (1:2,000; cat. no. sc-93; Santa Cruz Biotechnology, Inc.), p-ERK1/2 (1:2,000; cat. no. sc-16982-R; Santa Cruz Biotechnology, Inc.) or GAPDH (1:5,000; cat. no. 10494-1-AP; ProteinTech Group, Inc., Chicago, IL, USA) at $4^{\circ} \mathrm{C}$ overnight. The following day, the membranes were washed with TBST three times and further incubated with a horseradish peroxidase (HRP)-conjugated secondary antibody (1:1,000; cat. no. A0208; Beyotime Institute of Biotechnology) at $37^{\circ} \mathrm{C}$ for $2 \mathrm{~h}$. Finally, the protein bands were detected using an enhanced chemiluminescence reagent (Pierce; Thermo Fisher Scientific, Inc.). Densitometry was performed using Quantity one v4.6.7 software (Bio-Rad Laboratories, Inc., Hercules, CA, USA).
Knockdown of CXCR4. A lentivirus-based CXCR4 shRNA vector was constructed as previously described (29). A total of three types of CXCR4 small interfering (si)RNA were designed and chemically synthesized by Shanghai SunBio Biotechnology Co., Ltd. (Shanghai, China). The sequences were as follows: CXCR4 siRNA 1, 5'-GGAUAACUACUCCGAAGAAdTdT-3'; CXCR4 siRNA 2, 5'-CCAACAAGGAACCCTGCTTdTdT-3'; and CXCR4 siRNA 3, 5'-CCCTCAAGACTACGGTCATdTdT-3'. Following transfection into OPCs using Lipofectamine 2000 (Invitrogen; Thermo Fisher Scientific, Inc.), CXCR4 siRNA 2 exhibited the best efficiency at downregulating the expression of CXCR4. As a result, complementary DNA oligonucleotides of CXCR4 siRNA 2 were subcloned into a lentiviral vector to construct CXCR4 shRNA. A vector containing a scrambled sequence served as a CXCR4 shRNA control. Finally, OPCs were transfected with CXCR4 shRNA or shRNAcon for $72 \mathrm{~h}$ and subjected to western blot analysis or a migration assay. Based on the sequence of 5'-GCAAGAUCACACACCUCAUdTdT-3', siRNA of atypical chemokine receptor 3 (CXCR7) was chemically synthesized and subcloned into shRNA. The knockdown of CXCR7 was performed as described above.

Statistical analysis. Experimental results are presented as the mean \pm standard deviation and were analyzed using SPSS 19.0 statistical software (IBM Corp., Armonk, NY, USA) with one-way analysis of variance (ANOVA). Following ANOVA, the least significant difference post hoc test was used. Experiments were repeated three times. $\mathrm{P}<0.05$ was considered to indicate a statistically significant difference.

\section{Results}

Isolation, culturing and identification of rat OPCs. The present study isolated primary rat OPCs, as previously 

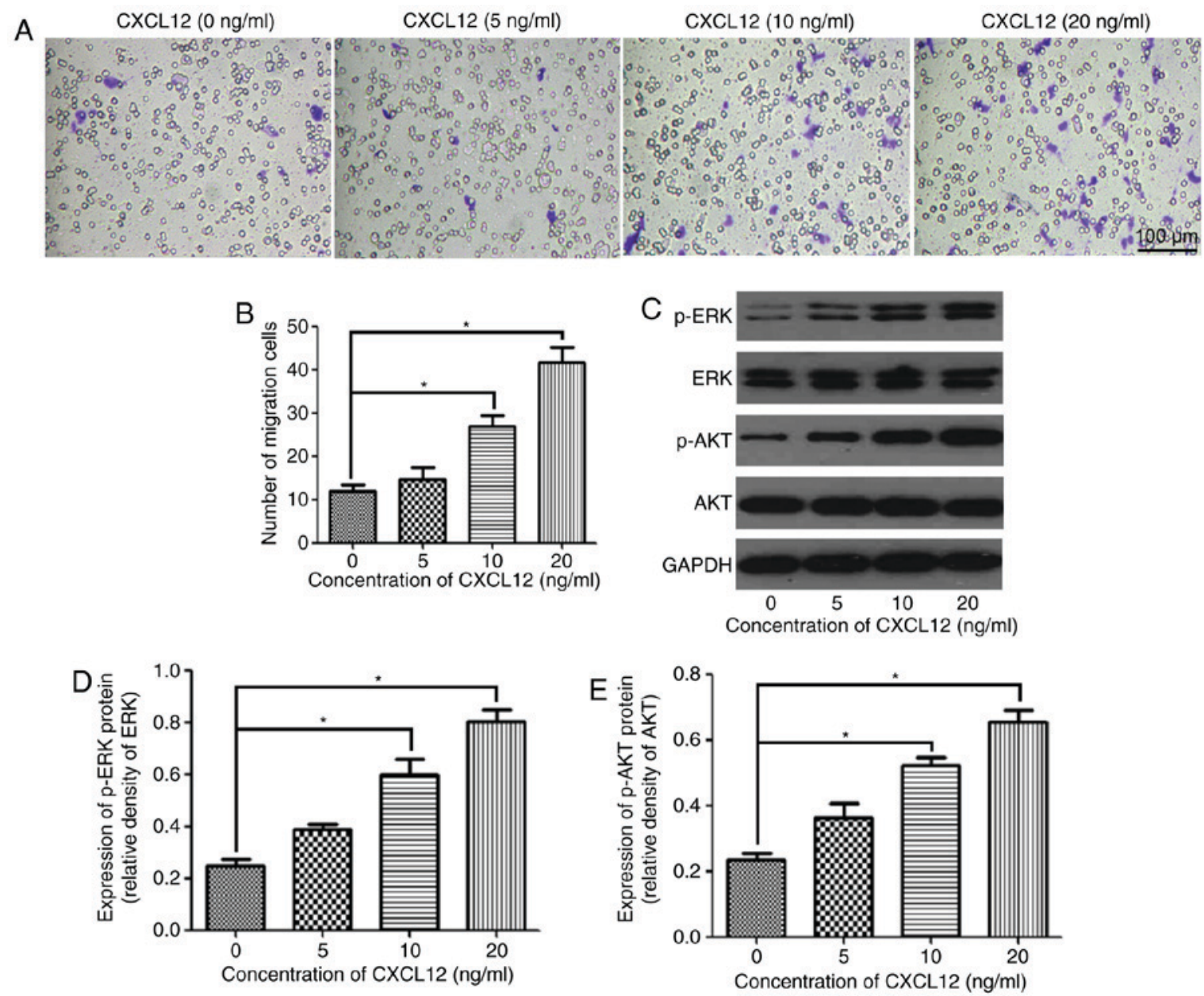

Figure 2. CXCL12 promotes the migration of OPCs. (A) Transwell migration assay of OPCs treated with different concentrations of CXCL12 for $48 \mathrm{~h}$. (B) Analysis of the data in each group. (C) Western blot analysis demonstrated the expression of p-ERK, ERK, p-AKT and AKT in the OPCs treated with different concentrations of CXCL12 for $48 \mathrm{~h}$. GAPDH served as a loading control. (D) Group data for the relative expression of p-ERK. (E) Group data for the relative expression of $\mathrm{p}-\mathrm{AKT}$. Data are presented as the mean \pm standard deviation. $\mathrm{n}=3$. "P<0.05. CXCL12, C-X-C motif chemokine ligand 12 ; OPCs, oligodendrocyte precursor cells; $p$, phosphorylated; ERK, extracellular signal-regulated kinase; AKT, RAC- $\alpha$ serine/threonine-protein kinase.

described $(27,28)$. The isolated cells harbored the typical morphology of OPCs, exhibiting a round body with bipolar and tripolar processes. The immunostaining assay confirmed that the isolated OPCs expressed NG2 and PDGFR- $\alpha$, OPC-specific markers (Fig. 1A and B), although they were negative for $\mathrm{O} 4$ and MBP, as markers of mature oligodendrocytes (data not shown). Following culturing in differentiation medium for 3 days, the OPCs were differentiated into mature oligodendrocytes, which highly expressed O4 and MBP (Fig. 1C and D). These findings confirmed that the isolated cells acquired the OPC phenotype.

CXCL12 induces the migration of OPCs. The present study assessed the effect of CXCL12 on the migration of OPCs using the Boyden chamber assay. The migration of OPCs was gradually enhanced with the increasing concentration of CXCL12 (Fig. 2A). According to the statistical analysis, the migration of OPCs was significantly increased by the chemoattractant effects of 10 and $20 \mathrm{ng} / \mathrm{ml}$ CXCL12 compared with the control group (0 $\mathrm{ng} / \mathrm{ml} \mathrm{CXCL12)} \mathrm{(Fig.} \mathrm{2B).} \mathrm{To} \mathrm{further} \mathrm{investigate} \mathrm{the}$ downstream mechanism of the CXCL12-induced migration of OPCs, the present study examined the expression of p-ERK and p-AKT, and demonstrated that p-ERK and p-AKT were significantly upregulated following treatment with 10 and $20 \mathrm{ng} / \mathrm{ml}$
CXCL12 (Fig. 2C-E). However, treatment with CXCL12 did not markedly affect the expression of ERK and AKT in OPCs (Fig. 2C-E). These findings suggested that the MEK/ERK and PI3K/AKT pathways are likely to be the downstream mechanism through which CXCL12 induces the migration of OPCs.

Knockdown of CXCR4 inhibits the migration of OPCs. Additionally, the effect of knockdown of CXCR4 on the CXCL12-induced migration of OPCs was assessed. A total of three types of CXCR4 siRNA were designed to knock down the expression of CXCR4, and CXCR4 siRNA 2 exhibited the best efficiency at downregulating the expression of CXCR4 (Fig. 3A and B). CXCR4 shRNA was constructed based on CXCR 4 siRNA 2. Under the chemotactic effects of $20 \mathrm{ng} / \mathrm{ml}$ CXCL12, the migration of CXCR4 shRNA-transfected OPCs was significantly decreased compared with the untreated and shRNAcon-treated OPCs (Fig. 3C and D). However, knockdown of CXCR7 (another receptor of CXCL12) did not affect CXCL12-induced migration (data not shown). In addition, CXCR4 shRNA significantly downregulated the expression of CXCR4 following treatment with $20 \mathrm{ng} / \mathrm{ml}$ CXCL12 (Fig. 3E and F). Notably, the expression of p-ERK and p-AKT was also downregulated by treatment with CXCR4 
A

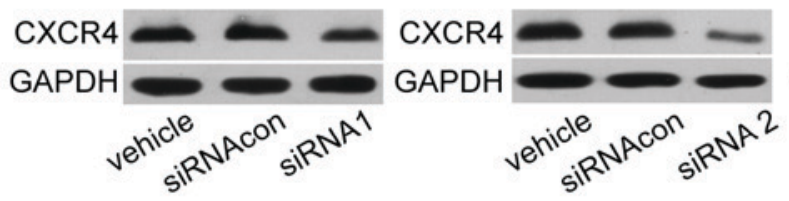

C

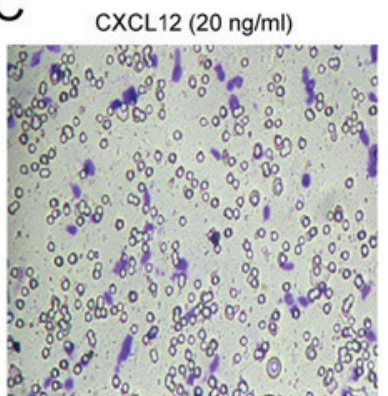

E

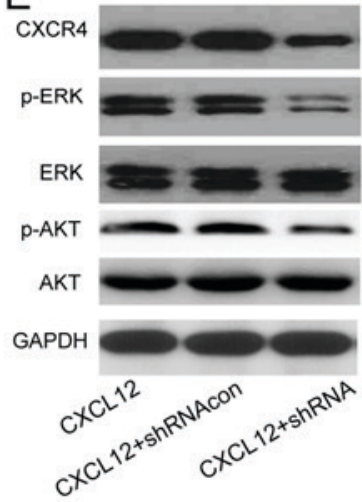

CXCL12 $(20 \mathrm{ng} / \mathrm{ml})+$ shRNAcon

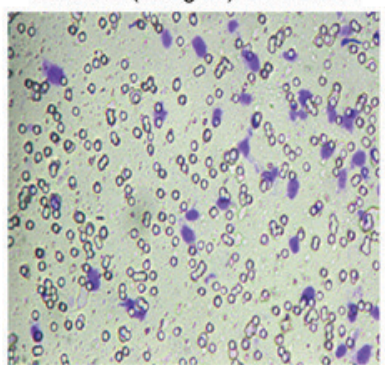

$\mathrm{F}$

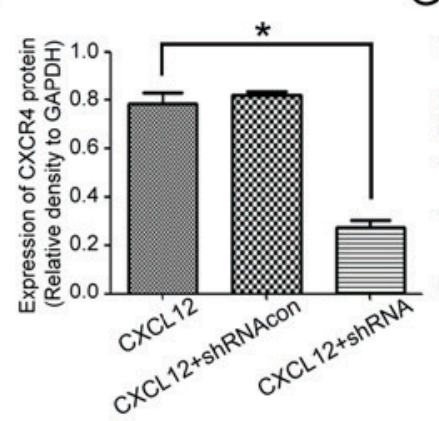

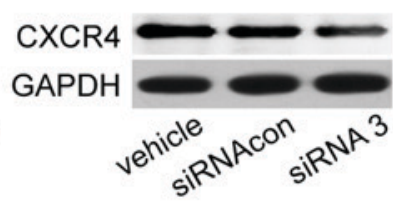

CXCL12 $(20 \mathrm{ng} / \mathrm{ml})+\operatorname{shRNA}$

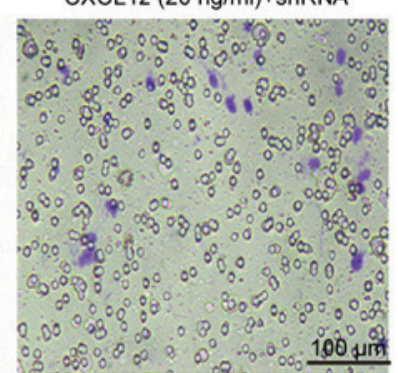

G

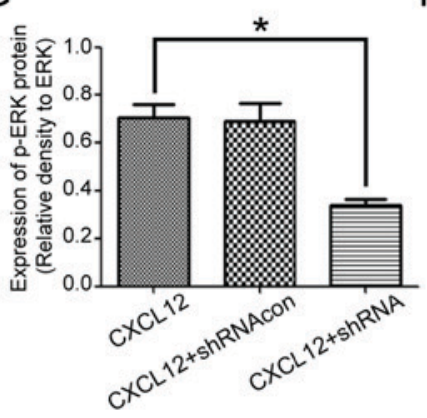

B
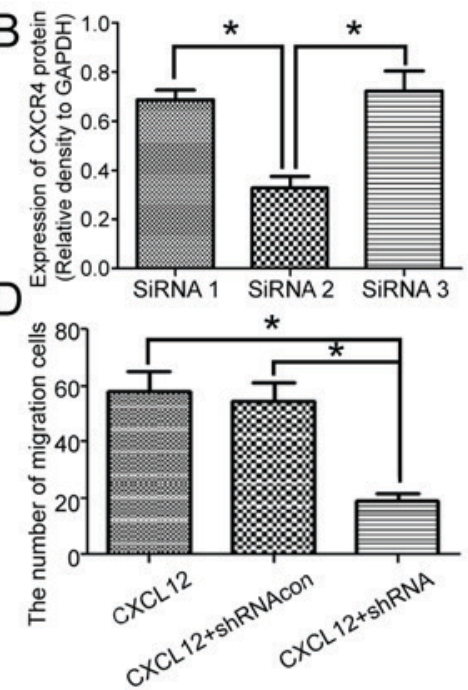

$\mathrm{H}$

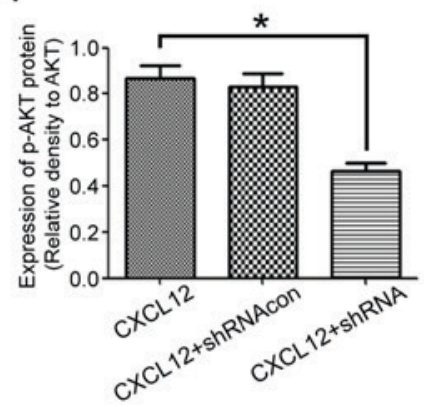

Figure 3. Knockdown of CXCR4 inhibits the CXCL12-induced migration of OPCs. (A) Western blot analysis demonstrated the expression of CXCR4 in the OPCs treated with vehicle (PBS), siRNAcon and each siRNA (siRNA1, siRNA2 and siRNA3). (B) Relative expression of CXCR4 in the OPCs treated with siRNA1, siRNA2 and siRNA3. (C) Transwell migration assay of OPCs treated with CXCL12 (20 ng/ml) and CXCR4 shRNA or shRNAcon. (D) Analysis of the migration data for each group. (E) Western blot analysis demonstrated the expression of CXCR4, p-ERK, ERK, p-AKT and AKT in the OPCs treated with CXCL12 (20 ng/ml) and CXCR4 shRNA or shRNAcon. GAPDH served as a loading control. (F) Group data for the relative expression of CXCR4. (G) Group data for the relative expression of p-ERK. (H) Group data for the relative expression of $\mathrm{p}-\mathrm{AKT}$. Data are presented as the mean \pm standard deviation. $\mathrm{n}=3$. ${ }^{*} \mathrm{P}<0.05$. CXCL12, C-X-C motif chemokine ligand 12; CXCR4, C-X-C motif chemokine receptor 4; siRNA, small interfering RNA; con, control; shRNA, short hairpin RNA; OPCs, oligodendrocyte precursor cells; p, phosphorylated; ERK, extracellular signal-regulated kinase; AKT, RAC- $\alpha$ serine/threonine-protein kinase.

shRNA, which supported the hypothesis that the MEK/ERK and PI3K/AKT pathways may be downstream of CXCR4 in OPCs (Fig. 3G and H). These data demonstrated that CXCL12 induced the migration of OPCs via CXCR4.

CXCL12-induced migration of OPCs is regulated by the $M E K / E R K$ and PI3K/AKT pathways. To further confirm that the MEK/ERK and PI3K/AKT pathways were involved in the CXCL12-induced migration of OPCs, U1026 and LY294002 were used as specific kinase inhibitors of the MEK/ERK and PI3K/AKT pathways to respectively block each signaling pathway. As expected, treatment with U1026 or LY294002 was able to significantly inhibit the CXCL12-induced migration of OPCs (Fig. 4A and B). Furthermore, western blot analysis demonstrated that U1026 specifically downregulated the expression of p-ERK in OPCs following treatment with 20 ng/ml CXCL12 (Fig. 4C and D). Likewise, LY294002 specifically downregulated the expression of p-AKT in OPCs (Fig. 4C and E). Taken together, these results suggested that the CXCL12-induced migration of OPCs was regulated by the MEK/ERK and PI3K/AKT pathways.

\section{Discussion}

Investigating the migration of OPCs is of great importance to improve remyelination in the CNS. The results of the present study demonstrated that CXCL12 induced the migration of OPCs via the CXCR4-activated MEK/ERK and PI3K/AKT pathways.

According to a well-established protocol, neonatal OPCs were separated by shaking and differential adhesion $(27,28)$. The obtained OPCs were positive for NG2 and PDGFR- $\alpha$ and were able to differentiate into mature oligodendrocytes, which were positive for $\mathrm{O} 4$ and MBP. In addition, it was observed that 10 and $20 \mathrm{ng} / \mathrm{ml} \mathrm{CXCL12} \mathrm{significantly} \mathrm{promoted} \mathrm{the} \mathrm{migra-}$ tion of OPCs in vitro, which is consistent with the results of a previous study (22). By contrast, another study demonstrated that CXCL12 inhibits the migration of OPCs and augments the differentiation of OPCs into mature oligodendrocytes (30). It has been reported that the expression of CXCR4 decreases gradually during the differentiation of OPCs (22). CXCR4, as a receptor of CXCL12, serves an important role in the migration of OPCs. As a result, when CXCL12 induced the differentiation 

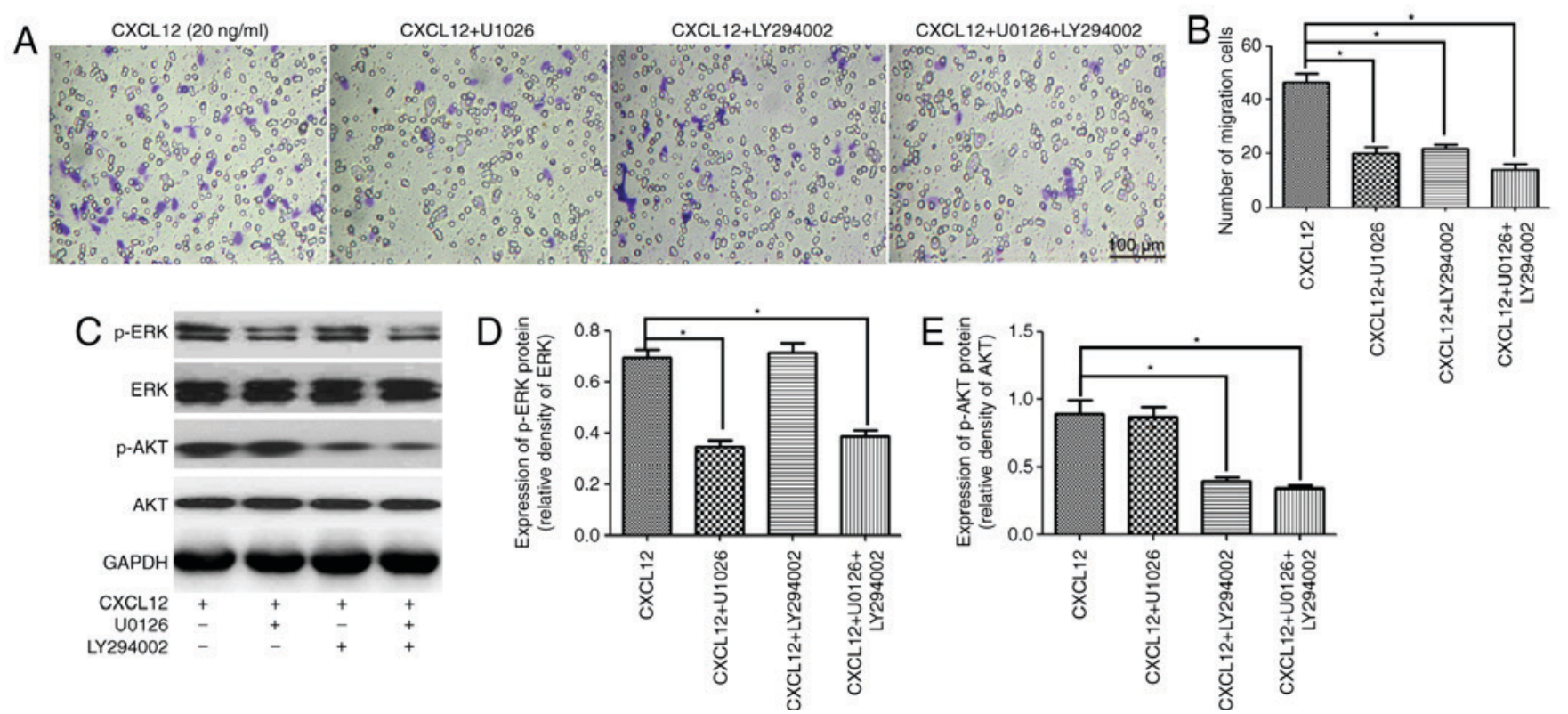

Figure 4. MEK/ERK and PI3K/AKT pathways are involved in the CXCL12-induced migration of OPCs. (A) Transwell migration assay of OPCs treated with CXCL12 $(20 \mathrm{ng} / \mathrm{ml})$ and an inhibitor of the MEK/ERK pathway (U1026), or an inhibitor of the PI3K/AKT pathway (LY294002). (B) Group migration data. (C) Western blot analysis demonstrated the expression of p-ERK, ERK, p-AKT and AKT in the OPCs treated with CXCL12 (20 ng/ml) and U1026 or LY294002. GAPDH served as a loading control. (D) Group data for the relative expression of p-ERK. (E) Group data for the relative expression of p-AKT. Data are presented as the mean \pm standard deviation. $\mathrm{n}=3$. ${ }^{*} \mathrm{P}<0.05$. CXCL12, C-X-C motif chemokine ligand 12; OPCs, oligodendrocyte precursor cells; $\mathrm{p}$, phosphorylated; ERK, extracellular signal-regulated kinase; AKT, RAC- $\alpha$ serine/threonine-protein kinase; MEK, dual specificity mitogen-activated protein kinase kinase 1; PI3K, phosphoinositide 3-kinase.

of OPCs, CXCL12 was insufficient in promoting the migration of OPCs due to the downregulation of CXCR4. However, CXCL12 promoted the migration of OPCs in the present study as the OPCs were prevented from differentiating in the proliferation medium. In addition, it was additionally demonstrated that knockdown of CXCR4 inhibited the CXCL12-induced migration of OPCs in vitro. Dziembowska et al (22) also demonstrated that $\mathrm{CXCR}^{-/-}$mice exhibit defective migration of OPCs in vivo. This suggests that CXCL12 may induce the migration of OPCs through CXCR4.

Furthermore, the present study demonstrated that the MEK/ERK and PI3K/AKT pathways were downstream of CXCL12/CXCR4. Treatment with CXCL12 was able to activate the MEK/ERK and PI3K/AKT pathways, while knockdown of CXCR4 inhibited the MEK/ERK and PI3K/AKT pathways. Using high-throughput quantitative phosphoproteomic analysis, Yi et al (31) demonstrated that the MEK/ERK pathway is downstream of CXCL12/CXCR4 in breast cancer stem cells. Notably, specific inhibitors of the MEK/ERK and PI3K/AKT pathways significantly reduced the migration of OPCs, which supported the hypothesis that the MEK/ERK and PI3K/AKT pathways were involved in the migration of OPCs. This finding was in agreement with previous results demonstrating that the CXCL12/CXCR4-activated MEK/ERK and $\mathrm{PI} 3 \mathrm{~K} / \mathrm{AKT}$ pathways regulated the migration of cancer cells $(24-26,32,33)$. Considering the fact that CXCL12/CXCR4 increases the phosphorylation of a number of cell migration- and invasion-associated proteins in breast cancer stem cells (31), there may be other pathways involved in the CXCL12/CXCR4-induced migration of OPCs.

Taken together, the results of the present study confirmed that CXCL12 induces the migration of OPCs through the
CXCR4-activated MEK/ERK and PI3K/AKT pathways. This study provides an experimental basis for the improved understanding of the CXCL12-induced migration of OPCs, which is of translational importance in improving remyelination.

\section{Acknowledgements}

Not applicable.

\section{Funding}

The present study was supported by the National Natural Science Foundation of China (grant no. 81471262).

\section{Availability of data and materials}

The datasets used and/or analyzed during the current study are available from the corresponding author on reasonable request.

\section{Authors' contributions}

XR and TJ conceived and designed the experiments. YT, XD and BT performed the experiments. YT and HY analyzed the data. XR and TJ contributed reagents and materials. HY and TJ wrote the paper.

\section{Ethics approval and consent to participate}

All procedures were performed according to protocols approved by the Institutional Review Board of Third Military Medical University and conformed to the National Institutes 
of Health (Bethesda, MD, USA) guide for the care and use of laboratory animals.

\section{Patient consent for publication}

Not applicable.

\section{Competing interests}

The authors declare that they have no competing interests.

\section{References}

1. Nave K: Myelination and the trophic support of long axons. Nat Rev Neurosci 11: 275-283, 2010.

2. Franklin RJM and ffrench-Constant C: Regenerating CNS myelin-from mechanisms to experimental medicines. Nat Rev Neurosci 18: 753-769, 2017

3. Young KM, Psachoulia K, Tripathi RB, Dunn SJ, Cossell L, Attwell D, Tohyama K and Richardson WD: Oligodendrocyte dynamics in the healthy adult CNS: Evidence for myelin remodeling. Neuron 77: 873-885, 2013.

4. De La Fuente AG, Lange S, Silva ME, Gonzalez GA, Tempfer H, van Wijngaarden P, Zhao C, Di Canio L, Trost A, Bieler L, et al: Pericytes stimulate oligodendrocyte progenitor cell differentiation during CNS remyelination. Cell Rep 20: 1755-1764, 2017.

5. Guo YE, Suo N, Cui X, Yuan Q and Xie X: Vitamin C promotes oligodendrocytes generation and remyelination. Glia 66 1302-1316, 2018.

6. Alizadeh A, Dyck SM and Karimi-Abdolrezaee S: Myelin damage and repair in pathologic CNS: Challenges and prospects. Front Mol Neurosci 8: 35, 2015.

7. Tokunaga H, Seiwa C, Yoshioka N, Mizoguchi K, Yamamoto M, Asou $\mathrm{H}$ and Aiso S: An extract of chinpi, the dried peel of the citrus fruit unshiu, enhances axonal remyelination via promoting the proliferation of oligodendrocyte progenitor cells. Evid Based Complement Alternat Med 2016: 8692698, 2016.

8. Ossola B, Zhao C, Compston A, Pluchino S, Franklin RJM and Spillantini MG: Neuronal expression of pathological tau accelerates oligodendrocyte progenitor cell differentiation. Glia 64: 457-471, 2016.

9. Huang S, Tang C, Sun S, Cao W, Qi W, Xu J, Huang J, Lu W, Liu Q, Gong B, et al: Protective effect of electroacupuncture on neural myelin sheaths is mediated via promotion of oligodendrocyte proliferation and inhibition of oligodendrocyte death after compressed spinal cord injury. Mol Neurobiol 52: 1870-1881, 2015 .

10. Hackett AR, Lee DH, Dawood A, Rodriguez M, Funk L, Tsoulfas P and Lee JK: STAT3 and SOCS3 regulate NG2 cell proliferation and differentiation after contusive spinal cord injury. Neurobiol Dis 89: 10-22, 2016.

11. Chen LX, Ma SM, Zhang P, Fan ZC, Xiong M, Cheng GQ, Yang Y, Qiu ZL, Zhou WH and Li J: Neuroprotective effects of oligodendrocyte progenitor cell transplantation in premature rat brain following hypoxic-ischemic injury. PLoS One 10 : e0115997, 2015.

12. Wang S, Bates J, Li X, Schanz S, Chandler-Militello D, Levine C, Maherali N, Studer L, Hochedlinger K, Windrem $M$ and Goldman SA: Human iPSC-derived oligodendrocyte progenitor cells can myelinate and rescue a mouse model of congenital hypomyelination. Cell Stem Cell 12: 252-264, 2013.

13. Yang J, Xiong LL, Wang YC, He X, Jiang L, Fu SJ, Han XF, Liu J and Wang TH: Oligodendrocyte precursor cell transplantation promotes functional recovery following contusive spinal cord injury in rats and is associated with altered microRNA expression. Mol Med Rep 17: 771-782, 2018.

14. Wu B, Sun L, Li P, Tian M, Luo Y and Ren X: Transplantation of oligodendrocyte precursor cells improves myelination and promotes functional recovery after spinal cord injury. Injury 43 : 794-801, 2012.
15. Gupta N, Henry RG, Strober J, Kang SM, Lim DA, Bucci M, Caverzasi E, Gaetano L, Mandelli ML, Ryan T, et al: Neural stem cell engraftment and myelination in the human brain. Sci Transl Med 4: 155ra137, 2012.

16. Boyd $\mathrm{A}$, Zhang $\mathrm{H}$ and Williams $\mathrm{A}$ : Insufficient $\mathrm{OPC}$ migration into demyelinated lesions is a cause of poor remyelination in MS and mouse models. Acta Neuropathol 125: 841-859, 2013.

17. Janssens R, Struyf S and Proost P: The unique structural and functional features of CXCL12. Cell Mol Immunol: Oct 30, 2017. (Epub ahead of print).

18. Meng W, Xue S and Chen Y: The role of CXCL12 in tumor microenvironment. Gene 641: 105-110, 2018.

19. Kadi L, Selvaraju R, de Lys P, Proudfoot AE, Wells TN and Boschert U: Differential effects of chemokines on oligodendrocyte precursor proliferation and myelin formation in vitro. J Neuroimmunol 174: 133-146, 2006.

20. Patel JR, McCandless EE, Dorsey D and Klein RS: CXCR4 promotes differentiation of oligodendrocyte progenitors and remyelination. Proc Natl Acad Sci USA 107: 11062-11067, 2010.

21. Zilkha-Falb R, Kaushansky N, Kawakami N and Ben-Nun A Post-CNS-inflammation expression of CXCL12 promotes the endogenous myelin/neuronal repair capacity following spontaneous recovery from multiple sclerosis-like disease. J Neuroinflammation 13: 7, 2016.

22. Dziembowska M, Tham T, Lau P, Vitry S, Lazarini $F$ and Dubois-Dalcq M: A role for CXCR4 signaling in survival and migration of neural and oligodendrocyte precursors. Glia 50: 258-269, 2005.

23. Carbajal KS, Miranda JL, Tsukamoto MR and Lane TE: CXCR4 signaling regulates remyelination by endogenous oligodendrocyte progenitor cells in a viral model of demyelination. Glia 59: 1813-1821, 2011.

24. Sobolik T, Su YJ, Wells S, Ayers GD, Cook RS and Richmond A: CXCR4 drives the metastatic phenotype in breast cancer through induction of CXCR2 and activation of MEK and PI3K pathways. Mol Biol Cell 25: 566-582, 2014.

25. Kukreja P, Abdel-Mageed AB, Mondal D, Liu K and Agrawal KC: Up-regulation of CXCR4 expression in PC-3 Cells by stromal-derived factor-1 $\alpha$ (CXCL12) increases endothelial adhesion and transendothelial migration: Role of MEK/ERK signaling pathway-dependent NF-kappaB activation. Cancer Res 65: 9891-9898, 2005.

26. Huang CY, Lee CY, Chen MY, Yang WH, Chen YH, Chang CH, Hsu HC, Fong YC and Tang CH: Stromal cell-derived factor-1/CXCR4 enhanced motility of human osteosarcoma cells involves MEK1/2, ERK and NF-kB-dependent pathways. J Cell Physiol 221: 204-212, 2009.

27. Armstrong R: Isolation and characterization of immature oligodendrocyte lineage cells. Armstrong RC 16: 282-292, 1998.

28. Itoh K: Culture of oligodendrocyte precursor cells (NG2+/O1-) and oligodendrocytes (NG2(-)/O1(+)) from embryonic rat cerebrum. Brain Res Brain Res Protoc 10: 23-30, 2002

29. Yu X, Chen D, Zhang Y, Wu X, Huang Z, Zhou H, Zhang Y and Zhang Z: Overexpression of CXCR4 in mesenchymal stem cells promotes migration, neuroprotection and angiogenesis in a rat model of stroke. J Neurol Sci 316: 141-149, 2012.

30. Maysami S, Nguyen D, Zobel F, Pitz C, Heine S, Höpfner M and Stangel M: Modulation of rat oligodendrocyte precursor cells by the chemokine CXCL12. Neuroreport 17: 1187-1190, 2006.

31. Yi T, Zhai B, Yu Y, Kiyotsugu Y, Raschle T, Etzkorn M, Seo HC, Nagiec M, Luna RE, Reinherz EL, et al: Quantitative phosphoproteomic analysis reveals system-wide signaling pathways downstream of SDF-1/CXCR4 in breast cancer stem cells. Proc Natl Acad Sci USA 111: E2182-E2190, 2014.

32. Yu T, Wu Y, Helman JI, Wen Y, Wang C and Li L: CXCR4 promotes oral squamous cell carcinoma migration and invasion through inducing expression of MMP-9 and MMP-13 via the ERK signaling pathway. Mol Cancer Res 9: 161-172, 2011.

33. Sun X, Wei L, Chen Q and Terek R: CXCR4/SDF1 mediate hypoxia induced chondrosarcoma cell invasion through ERK signaling and increased MMP1 expression. Mol Cancer 9: 17, 2010.

This work is licensed under a Creative Commons Attribution 4.0 International (CC BY 4.0) License. 